\title{
DEGREE OF APPROXIMATION TO FUNCTIONS ON A JORDAN CURVE
}

\author{
BY \\ J. L. WALSH
}

If $C$ is an arbitrary Jordan curve of the $z$-plane containing the origin in its interior, and if $f(z)$ is an arbitrary function continuous on $C$, it is known $[1$, p. 39] that $f(z)$ can be represented on $C$ by a uniformly convergent sequence of polynomials in $z$ and $1 / z$ of the form

$$
p_{n}(z) \equiv \sum_{k=-n}^{n} a_{n k} z^{k}
$$

Components of these functions are defined for $z$ interior to $C$ (assumed rectifiable) by the equations

$$
p_{1 n}(z) \equiv \sum_{k=0}^{n} a_{n k} z^{k} \equiv \frac{1}{2 \pi i} \int_{C} \frac{p_{n}(t) d t}{t-z}, \quad f_{1}(z) \equiv \frac{1}{2 \pi i} \int_{C} \frac{f(t) d t}{t-z},
$$

where the integrals are taken counterclockwise, and for $z$ exterior to $C$ by the equations

$$
p_{2 n}(z) \equiv \sum_{k=-n}^{-1} a_{n k} z^{k} \equiv \frac{1}{2 \pi i} \int_{C} \frac{p_{n}(t) d t}{t-z}, \quad f_{2}(z) \equiv \frac{1}{2 \pi i} \int_{C} \frac{f(t) d t}{t-z},
$$

where the integrals are taken clockwise; it follows that we have for $z$ interior to $C$

$$
\lim _{n \rightarrow \infty} p_{1 n}(z)=f_{1}(z)
$$

and for $z$ exterior to $C$

$$
\lim _{n \rightarrow \infty} p_{2 n}(z)=f_{2}(z) .
$$

It is the object of the present note to indicate that under suitable conditions (Theorem 1) various degrees of approximation of $p_{n}(z)$ to $f(z)$ on $C$ imply certain continuity properties of $f(z)$, and (Theorem 2 ) under suitable conditions equations (4) and (5) in more precise form are valid even on $C$ itself. We consider also (Theorems $3,4,5$ ) approximation on $C$ by analytic functions more general than polynomials $p_{n}(z)$, and prove the analogues of (4) and (5) in more precise form. We mention briefly (Theorems 6 and 7) approximation in a multiply-connected region.

Presented to the Society, February 23, 1952; received by the editors February 11, 1952. 
All numbers $A$ and $B$ with or without subscripts are constants independent of $n$ and $z$.

Theorem 1. Let $C$ be an analytic Jordan curve containing the origin in its interior, and let the function $f(z)$ be defined on $C$. A necessary and sufficient condition that $f(z)$ be of class $L(p, \alpha)$ for $0<\alpha<1$ or of class $Z_{p}$ for $\alpha=1$ on $C$ is that there exist polynomials $p_{n}(z)$ given by (1) such that we have $(n>0)$

$$
\left|f(z)-p_{n}(z)\right| \leqq A / n^{p+\alpha}, \quad z \text { on } C .
$$

Here $p$ is a non-negative integer and $L(p, \alpha)$ and $Z_{p}$ are respectively the classes of functions continuous on $C$ whose $p$ th derivatives with respect to arc length on $C$ are continuous and satisfy on $C$ a Lipschitz condition of order $\alpha$ or a condition of form

$$
|F(s+h)+F(s-h)-2 F(s)| \leqq A_{1}|h|
$$

uniformly with respect to arc length $s$ and $h$; this latter condition has been investigated especially by Zygmund.

The existence of the $p_{n}(z)$ satisfying (6) has already been established [2], by separate approximation in the two closed regions bounded by $C$ of the respective components of $f(z)$.

Conversely, if inequalities (6) are valid there exists some constant $A_{2}$ such that we have $\left|p_{n}(z)\right| \leqq A_{2}, z$ on $C$. In a suitably chosen annulus containing $C$ in its interior we then have $[1$, p. 259]

$$
\left|p_{n}(z)\right| \leqq A_{2} R^{n} \text {. }
$$

Inequalities (6) and (8) imply [3, Theorem 4] that $f(z)$ is of class $L(p, \alpha)$ if $0<\alpha<1$ and is of class $Z_{p}$ if $\alpha=1$ on each of two open Jordan subarcs of $C$ which overlap at both ends and cover $C$; this implies the conclusion of Theorem 1( $\left.{ }^{1}\right)$.

THEOREM 2. Let $C$ be an analytic Jordan curve containing the origin in its interior, and suppose for a sequence of polynomials $p_{n}(z)$ given by (1) and for some function $f(z)$ we have $(n>0)$

$$
\left|f(z)-p_{n}(z)\right| \leqq A_{2} / n^{p+\alpha}, \quad z \text { on } C,
$$

where $p$ is a non-negative integer, $0<\alpha \leqq 1$. Then in the notation of (2) and (3) we have, for a suitably chosen $A_{3},(n>1)$

$$
\begin{aligned}
& \left|f_{1}(z)-p_{1 n}(z)\right| \leqq A_{3} \log n / n^{p+\alpha}, \quad z \text { on } C, \\
& \left|f_{2}(z)-p_{2 n}(z)\right| \leqq A_{3} \log n / n^{p+\alpha}, \quad z \text { on } C .
\end{aligned}
$$

Several lemmas are convenient in the proof of Theorem 2.

(1) We remark incidentally that Theorem 1 and numerous other results [3] assuming various degrees of approximation can be extended to apply under the hypothesis of more general degrees of approximation, by application of classical theorems due to de la Vallee Poussin. 
Lемма 1. Let the function $F(z)$ be measurable and in modulus not greater than $M$ on $C:|z|=1$, with the formal development on $C$

$$
F(z) \sim \sum_{k=-\infty}^{\infty} a_{k} z^{k}, \quad a_{k}=\frac{1}{2 \pi i} \int_{C} \frac{F(z) d z}{z^{k+1}} .
$$

Then there exists an absolute constant $B$ such that $(n>1)$

$$
\left|\sum_{k=-n}^{n} a_{k} z^{k}\right| \leqq B \cdot M \cdot \log n, \quad z \text { on } C .
$$

On $C$ we write $z=e^{i \theta}$,

$$
\cos k \theta=\frac{z^{k}+z^{-k}}{2}, \quad \sin k \theta=\frac{z^{k}-z^{-k}}{2 i},
$$

so the summation in (12) is precisely the sum of the first $n+1$ terms of the formal Fourier expansion of $F(z)$; inequality (12) is merely another formulation of a well known inequality due to Lebesgue.

LEMMA 2. Under the hypothesis of Lemma 1, there exists an absolute constant $B^{\prime}$ such that

$$
\begin{aligned}
& \left|\sum_{k=0}^{n} a_{k} z^{k}\right| \leqq B^{\prime} M \log n, \quad z \text { on } C, \\
& \left|\sum_{k=-n}^{-1} a_{k} z^{k}\right| \leqq B^{\prime} M \log n, \quad \text { z on } C .
\end{aligned}
$$

We note that the formal expansion on $C$ of $z^{p} F(z)$ is

$$
z^{\nu} F(z) \sim \sum_{k=-\infty}^{\infty} a_{k} z^{k+\nu}
$$

for we have by (11)

$$
\frac{1}{2 \pi i} \int_{C} \frac{z^{\nu} F(z) d z}{z^{k+1}}=\frac{1}{2 \pi i} \int_{C} \frac{F(z) d z}{z^{k-\nu+1}}=a_{k-\nu} ;
$$

of course on $C$ we have $\left|z^{\nu} F(z)\right| \leqq M$, and we have $\left|a_{k}\right| \leqq M$. If $n(>2)$ is even there follows by applying Lemma 1 to the function $z^{-n / 2} F(z)$

$$
\left|\sum_{k=0}^{n} a_{k} z^{k}\right| \leqq B M \log (n / 2), \quad z \text { on } C ;
$$

if $n(>3)$ is odd we have by use of (13)

$$
\left|\sum_{k=0}^{n-1} a_{k} z^{k}+a_{n} z^{n}\right| \leqq B M \log [(n-1) / 2]+M, z \text { on } C .
$$


The conclusion of Lemma 2 now follows from (13), (14), similar inequalities for the case $n=2$ and $n=3$, and from (12).

Lemma 3. Let $C$ be an analytic Jordan curve containing the origin $z=0$ in its interior; there exists a constant $B^{\prime}(C)$ depending only on $C$ such that if $F(z) \equiv \sum_{k=-n}^{n} a_{k} z^{k}$ and if we have $|F(z)| \leqq M$ on $C$, then we have on $C(n>1)$

$$
\left|\sum_{k=0}^{n} a_{k} z^{k}\right| \leqq B^{\prime}(C) M \log n .
$$

Here it is convenient to use Faber's polynomials $\phi_{n}(z), n=0,1,2, \cdots$, of respective degrees $n$ belonging to $C$. Let $w=\phi(z), z=\psi(w)$ map the exterior of $C$ onto the exterior of $\gamma:|w|=1$ with $\phi(\infty)=\infty$. Then we have for $z$ interior to $C$ (the details are discussed in more detail in [2])

$$
\begin{aligned}
\sum_{k=0}^{n} a_{k} z^{k} & \equiv \frac{1}{2 \pi i} \int_{C} \frac{F(t) d t}{t-z} \equiv \sum_{k=0}^{n} c_{k} \phi_{k}(z), \\
c_{k} & =\frac{1}{2 \pi i} \int_{\gamma} \frac{F[\psi(w)] d w}{w^{k+1}} ;
\end{aligned}
$$

this expansion is valid also on $C$. It is to be noted that the coefficients $c_{k}$ of order greater than $n$ all vanish, for we have $(k>n)$

$$
\int_{\gamma} \frac{F[\psi(w)] d w}{w^{k+1}}=0
$$

since the integrand is analytic for $|w| \geqq 1$ and vanishes to at least the second order at infinity.

On $C$ we have $\phi_{k}(z) \equiv w^{k}+r_{k}(z)$, where $\left|r_{k}(z)\right| \leqq A^{\prime} r^{k}, 0<r<1, A^{\prime}$ and $r$ depend only on $C$, so we can write on $C$

$$
\sum_{k=0}^{n} c_{k} \phi_{k}(z) \equiv \sum_{k=0}^{n} c_{k} w^{k}+\sum_{k=0}^{n} c_{k} r_{k}(z)
$$

The first sum in the second member is on $\gamma$ precisely the first sum of Lemma 2; moreover we have $\left|c_{k}\right| \leqq M$, whence

$$
\left|\sum_{k=0}^{n} c_{k} r_{k}(z)\right| \leqq M A^{\prime} /(1-r) ;
$$

thus we have $(n>1)$

$$
\left|\sum_{k=0}^{n} c_{k} \phi_{k}(z)\right| \leqq\left[B^{\prime} \log n+A^{\prime} /(1-r)\right] M,
$$

from which Lemma 3 follows.

Of course the proof and conclusion of Lemma 3 apply under broader 
conditions than those mentioned; the conclusion essentially refers to the sum of the first $n+1$ terms of the formal development in Faber polynomials of an arbitrary measurable function on $C$ with bound $M$.

We are now in a position to complete the proof of Theorem 2. From (9) it follows by Theorem 1 that $f(z)$ is of class $L(p, \alpha)$ or $Z_{p}$ on $C$ according as we have $0<\alpha<1$ or $\alpha=1$. Consequently, by the theory of the boundary values of the functions represented by the Cauchy integral as first considered by Plemelj $[2 ; 7]$, it follows that $f_{1}(z)$ and $f_{2}(z)$ as defined by (2) and (3) are also of class $L(p, \alpha)$ or $Z_{p}$ on $C$. Then there exist [for $0<\alpha<1$ this result is due to Curtiss; see 2,4$]$ polynomials $P_{1 n}(z)$ in $z$ and $P_{2 n}(z)$ in $1 / z$ of degrees $n$ such that we have on $C(n>0)$

$$
\left|f_{1}(z)-P_{1 n}(z)\right| \leqq A_{4} / n^{p+\alpha}, \quad\left|f_{2}(z)-P_{2 n}(z)\right| \leqq A_{4} / n^{p+\alpha} .
$$

However, $f_{2}(z)$ vanishes at infinity and it is important to choose the polynomials $P_{2 n}(z)$ so as to vanish at infinity; this is done by choosing arbitrary polynomials of the requisite degree of convergence on $C$, a degree of convergence valid throughout the closed exterior of $C$ and hence at infinity, and subtracting from each approximating polynomial its value at infinity; such subtraction does not alter the degree of convergence on $C$ nor the function approximated, namely $f_{2}(z)$. In the notation of $(2)$ and (3) we write (9) in the form $\left|f_{1}(z)+f_{2}(z)-p_{1 n}(z)-p_{2 n}(z)\right| \leqq A_{2} / n^{p+\alpha}, z$ on $C$, which by (15) gives

$$
\left|P_{1 n}(z)+P_{2 n}(z)-p_{1 n}(z)-p_{2 n}(z)\right| \leqq A_{5} / n^{p+\alpha}, \quad z \text { on } C .
$$

Lemma 3 , together with a corresponding conclusion concerning $P_{2 n}(z)-p_{2 n}(z)$, -a direct consequence of hypothesis and conclusion of Lemma 3-now yields $(n>1)$ for $z$ on $C$

$$
\begin{aligned}
& \left|P_{1 n}(z)-p_{1 n}(z)\right| \leqq A_{6} \log n / n^{p+\alpha} \\
& \left|P_{2 n}(z)-p_{2 n}(z)\right| \leqq A_{6} \log n / n^{p+\alpha}
\end{aligned}
$$

and (10) follows from (15).

Theorem 2 assumes the validity of (9) for every $n(>0)$. It is readily shown by standard methods that the validity of (9) for an infinite sequence of indices not possessing arbitrarily large gaps implies the existence of $p_{n}(z)$ defined for every $n(>0)$ such that (9) is valid, after possible modification of the constant $A_{2}$. If the hypothesis of Theorem 2 is modified so as to assume that $f(z)$ is of class $L(p, \alpha)$ or $Z_{p}$ on $C$, and if (9) is assumed not for every $n$ but for an infinite sequence of indices $n$, then (10) is valid for that same sequence.

Of course Theorem 2 cannot be extended to the general case where we assume $p_{n}(z)$ merely to converge to $f(z)$ uniformly on $C$ and attempt to prove (4) and (5) uniformly on $C$, for if $f(z)$ is an arbitrary function continuous on $C$ there exists a sequence $p_{n}(z)$ converging to $f(z)$ uniformly on $C$, but the func- 
tions $f_{1}(z)$ and $f_{2}(z)$ defined by (2) and (3) need not be continuous on $C$. A sufficient condition that (4) and (5) hold uniformly on $C$ is that $p_{n}(z)$ converge to $f(z)$ on $C$ with a degree of convergence $1 / n^{\beta}$ for some $\beta>0$.

On the other hand $[1, \S 9.9]$ it is true that if we replace (9) by the hypothesis

$$
\limsup _{n \rightarrow \infty}\left[\max \left|f(z)-p_{n}(z)\right|, z \text { on } C\right]^{1 / n} \leqq 1 / R<1,
$$

the inequalities (10) may be replaced by the conclusion

$$
\begin{aligned}
& \limsup _{n \rightarrow \infty}\left[\max \left|f_{1}(z)-p_{1 n}(z)\right|, z \text { on } C\right]^{1 / n} \leqq 1 / R, \\
& \limsup _{n \rightarrow \infty}\left[\max \left|f_{2}(z)-p_{2 n}(z)\right|, z \text { on } C\right]^{1 / n} \leqq 1 / R .
\end{aligned}
$$

Theorem 2 is related to, and can be extended to include, approximation by functions more general than polynomials:

THEOREM 3. Let $C$ be an analytic Jordan curve contained in an annular region $D$. Let the sequence of functions $F_{n}(z)$ analytic in $D$ satisfy the inequality

$$
\left|F_{n}(z)\right| \leqq A_{7} R^{n}, \quad R>1, \quad z \text { in } D,
$$

and suppose for some function $f(z)$ defined on $C$ we have $(n>0)$

$$
\left|f(z)-F_{n}(z)\right| \leqq A_{8} / n^{p+\alpha}, \quad z \text { on } C .
$$

Then on $C$ the function $f(z)$ is of class $L(p, \alpha)$ if $0<\alpha<1$, and of class $Z_{p}$ if $\alpha=1$. Moreover, if we set as in (2) and (3)

$$
F_{1 n}(z) \equiv \frac{1}{2 \pi i} \int_{C} \frac{F_{n}(t) d t}{t-z}, \quad F_{2 n}(z) \equiv \frac{1}{2 \pi i} \int_{C} \frac{F_{n}(t) d t}{t-z},
$$

respectively for $z$ interior and exterior to $C$, then we have $(n>1)$

$$
\begin{aligned}
& \left|f_{1}(z)-F_{1 n}(z)\right| \leqq A_{9} \log n / n^{p+\alpha}, \text { z on } C, \\
& \left|f_{2}(z)-F_{2 n}(z)\right| \leqq A_{9} \log n / n^{p+\alpha}, \text { z on } C .
\end{aligned}
$$

Let $w=\phi(z)$ map the exterior of $C$ onto $|w|>1$ with $\phi(\infty)=\infty$; let $z=0$ lie interior to $C$, and let $w=\Phi(z)$ map the interior of $C$ onto $|w|<1$ with $\Phi(0)=0$. We assume the functions $F_{n}(z)$ to be analytic and to satisfy (17) in the closure of $D$, where $D$ is now bounded by the curve $C_{\rho}:|\phi(z)|=\rho(>1)$ exterior to $C$ and the curve $C_{1 / \rho}:|\Phi(z)|=1 / \rho$ interior to $C$.

We expand $F_{1 n}(z)$ in the Faber polynomials belonging to $C$, where the function is represented by the formula

$$
F_{1 n}(z) \equiv \frac{1}{2 \pi i} \int_{C_{\rho}} \frac{F_{n}(t) d t}{t-z}, \quad z \text { interior to } C_{\rho},
$$

and the coefficients are found by integration over $C_{\rho}$ or over $|w|=\rho$. Then 
there exist [compare 3] polynomials $p_{1, \lambda n}(z)$ in $z$ of respective degrees $\lambda n$ such that we have

$$
\left|F_{1 n}(z)-p_{1, \lambda n}(z)\right| \leqq B_{1}\left(R / \rho^{\lambda}\right)^{n}, \quad z \text { on } C ;
$$

here $\lambda$ is any positive integer such that $R / \rho^{\lambda}<1$.

In a precisely similar way, we expand $F_{2 n}(z)$ defined now by the formula

$$
F_{2 n}(z) \equiv \frac{1}{2 \pi i} \int_{C_{1 / \rho}} \frac{F_{n}(t) d t}{t-z}, \quad z \text { exterior to } C_{1 / \rho},
$$

in polynomials in $1 / z$, the expansion being valid throughout the closed exterior of $C_{1 / \rho}$. The function $F_{2 n}(z)$ vanishes at infinity and thus, as in the proof of Theorem 2 , for suitably chosen polynomials $p_{2, \lambda n}(z)$ in $1 / z$ of respective degrees $\lambda n$ which vanish at infinity we have

$$
\left|F_{2 n}(z)-p_{2, \lambda n}(z)\right| \leqq B_{2}\left(R / \rho^{\lambda}\right)^{n}, \quad z \text { on } C .
$$

The functions $f_{1}(z)$ and $f_{2}(z)$ represented by $(1)$ and $(2)$ are [3, Theorem 4 ] on $C$ of classes $L(p, \alpha)$ if $0<\alpha<1$ or $Z_{p}$ if $\alpha=1$. Consequently there exist polynomials $P_{1, \lambda n}(z)$ in $z$ of degrees $\lambda n$ and polynomials $P_{2, \lambda n}(z)$ in $1 / z$ of degrees $\lambda n$ vanishing at infinity such that we have $(n>0)$

$$
\begin{aligned}
& \left|f_{1}(z)-P_{1, \lambda n}(z)\right| \leqq B_{3} /(\lambda n)^{p+\alpha}, \quad z \text { on } C, \\
& \left|f_{2}(z)-P_{2, \lambda n}(z)\right| \leqq B_{3} /(\lambda n)^{p+\alpha}, \quad z \text { on } C .
\end{aligned}
$$

On $C$ the equations $f(z) \equiv f_{1}(z)+f_{2}(z), \quad F_{n}(z) \equiv F_{1 n}(z)+F_{2 n}(z)$ and inequalities (18), (21), (22), (23) yield $(n>0)$

$$
\left|P_{1, \lambda n}(z)+P_{2, \lambda n}(z)-p_{1, \lambda n}(z)-p_{2, \lambda n}(z)\right| \leqq B_{4} /(\lambda n)^{p+\alpha}, \quad z \text { on } C .
$$

From Lemma 3 we have $(n>1)$

$$
\begin{aligned}
& \left|P_{1, \lambda n}(z)-p_{1, \lambda n}(z)\right| \leqq B_{5} \log n / n^{p+\alpha}, \quad z \text { on } C, \\
& \left|P_{2, \lambda n}(z)-p_{2, \lambda n}(z)\right| \leqq B_{5} \log n / n^{p+\alpha}, \quad \dot{z} \text { on } C .
\end{aligned}
$$

Inequalities (20) now follow by (21), (22), and (23).

We add the remark that Theorem 3 is related to previous work [5], concerning approximation on an arbitrary rectifiable Jordan curve $C$ by certain functions $F_{n}(z)$ not required to be analytic on $C$ itself, and where we assume

$$
\limsup _{n \rightarrow \infty}\left[\max \left|f(z)-F_{n}(z)\right|, z \text { on } C\right]^{1 / n}<1 .
$$

Theorem 3 includes Theorem 2, for in the proof of Theorem 1 we have indicated that (6) implies (8), and (8) is precisely (17) for the functions $p_{n}(z)$. Still more can be concluded.

Lemma 4. Let $C$ be a Jordan curve, let $w=\phi(z)$ with $\phi(\infty)=\infty$ map the exterior of $C$ onto $|w|>1$, and let $C_{R}$ denote generically the Jordan curve 
$|\phi(z)|=R(>1)$ exterior to $C$. Let the function $R_{n}(z)$ be analytic exterior to $C$ and continuous in the corresponding closed region except possibly for poles not more than $n$ in number all of which lie in the closed exterior of $C_{\rho}$. Then lhe inequality

$$
\left|R_{n}(z)\right| \leqq L, \quad z \text { on } C
$$

implies $\left(R_{1}<\rho\right)$

$$
\left|R_{n}(z)\right| \leqq L\left(\frac{\rho R_{1}-1}{\rho-R_{1}}\right)^{n}, \text { z on } C_{R_{1}} .
$$

Lemma 4 can be proved by applying in the $w$-plane a known method of proof $[1$, p. 231, Lemma I].

THeOREM 4. Let $C$ be an analytic Jordan curve, let $F_{n}(z)$ be a sequence of rational functions of respective degrees $n$ whose poles have no limit point on $C$, and suppose for some function $f(z)$ defined on $C$ we have (18). Then we also have (17) for a suitably chosen $D$ and $R$; the conclusion of Theorem 3 is valid.

Since the poles of the $F_{n}(z)$ have no limit point on $C$, all such poles lie exterior to some annular region $D^{\prime}$ containing $C$ bounded by the two Jordan curves $|\phi(z)|=\rho$ and $|\Phi(z)|=1 / \rho$, where we assume the origin to lie interior to $C$ and $w=\Phi(z)$ maps the interior of $C$ onto $|w|<1$ with $\Phi(0)=0$. It follows from (18) that the functions $F_{n}(z)$ are uniformly bounded on $C$, and follows from Lemma 4 as applied first directly and second after map of the interior of $C$ onto $|w|>1$ that (17) holds with $R=\left(\rho R_{1}-1\right) /\left(\rho-R_{1}\right)$ in the annular region $D$ containing $C$ bounded by the two Jordan curves $|\phi(z)|=R_{1}$ $\left(1<R_{1}<\rho\right)$ exterior to $C$ and $|\Phi(z)|=1 / R_{1}$ interior to $C$. Thus the conclusion of Theorem 3 is valid. The first part of this conclusion, relative to the properties of $f(z)$ on $C$, has connections with recent results due to H. M. Elliott [6].

Like Theorem 2, the conclusion of Theorem 4 is valid if we assume (18) not for all $n(>0)$ but merely for an infinite sequence of indices $n$ which does not possess arbitrarily large gaps.

Theorem 3 has application to approximation by bounded analytic functions:

THEOREM 5. Let $C$ be an analytic Jordan curve containing the origin $O$ in its interior, and let $D$ be a bounded region containing $C$ whose closure does not contain $O$. Let the function $f(z)$ be continuous on $C$. For each positive $M$ let $F_{M}(z)$ be the (or a) function analytic and of modulus not greater than $M$ in $D$ such that the measure of approximation

$$
\mu_{M}=\max \left[\left|f(z)-F_{M}(z)\right|, z \text { on } C\right]
$$

is least. Then a necessary and sufficient condition that $f(z)$ be of class $L(p, \alpha)$ 
with $0<\alpha<1$ or of class $Z_{p}$ with $\alpha=1$ on $C$ is that

$$
\log M \cdot \mu_{M}^{1 /(p+\alpha)}
$$

be bounded as $M \rightarrow \infty$.

We merely sketch the proof of Theorem 5 , for the details are similar to those in the proof of an analogous result [3, Theorem 3]. If (25) is bounded, we choose the sequence of values $M=e^{n}$, and the conclusion follows from Theorem 3. Conversely, if $f(z)$ is given with the required properties on $C$, we make use of the polynomials of Theorem 1 . Inequality (8) is then valid in $D$, if $R$ is suitably chosen [1, p. 259], and the boundedness of (25) follows.

It may be noted that if we consider a sequence of extremal functions $F_{M}(z)$ for values $M=A R^{n}, R>1$, it follows from the boundedness of (25) that the analogue of (18) is valid, and hence the analogue of (20) is also valid.

If $f(z)$ is continuous on $C$ we introduce the definition

$$
\liminf _{M \rightarrow \infty}\left[\frac{-\log \mu_{M}}{\log \log M}\right]=\beta ;
$$

the values $\beta=+\infty$ and $\beta=-\infty$ are not excluded. Then whenever we have $p+\alpha<\beta$, the function $f(z)$ is of class $L(p, \alpha)$ or $Z_{p}$ on $C$ according as $0<\alpha<1$ or $\alpha=1$; but $f(z)$ is of no class $L(p, \alpha)$ or $Z_{p}$ on $C$ if we have $\beta \leqq 0$, and is not of the class $L(p, \alpha)$ or $Z_{p}$ on $C$ if we have $p+\alpha>\beta$. If $f(z)$ and $\beta$ are given, and if we have $p+\alpha<\beta$, then we have for $M$ sufficiently large

$$
\frac{-\log \mu_{M}}{\log \log M}>p+\alpha,
$$

from which the boundedness of (25) follows. Conversely, if $f(z)$ is of class $L(p, \alpha)$ or $Z_{p}$, the boundedness of (25) implies

$$
\begin{gathered}
\log \mu_{M}+(p+\alpha) \log \log M<B_{6}, \\
p+\alpha \leqq \beta .
\end{gathered}
$$

If $g_{M}(z)$ is an arbitrary function (not necessarily extremal) analytic and of modulus not greater than $M$ in $D$ we set

$$
\stackrel{*}{\mu_{M}}=\max \left[\left|f(z)-g_{M}(z)\right|, z \text { on } C\right],
$$

whence $\mu_{M}^{*} \geqq \mu_{M}$,

$$
\liminf _{M \rightarrow \infty}\left[\frac{-\log \mu_{M}^{*}}{\log \log M}\right] \leqq \beta .
$$

We emphasize the fact that this relation holds for an arbitrary family of functions $g_{M}(z)$ analytic and of modulus not greater than $M$ in $D$, and (as is 
readily shown) holds even if $M$ becomes infinite merely taking the values of a sequence $M=B_{0} R_{1}^{n}, R_{1}>1$.

We turn now from the study of approximation on a Jordan curve to the study of approximation on an annulus or region of higher connectivity, for the methods just developed apply in this new situation with relatively little change.

Theorem 6. Let $C$ be a closed point set whose boundary consists of an analytic Jordan curve $C_{0}$ and mutually exterior analytic Jordan curves $C_{1}, C_{2}, \cdots, C_{\nu}$ interior to $C_{0}$. Let $D_{k}$ denote that region of the extended plane bounded by $C_{k}$ containing the interior of $C$, and let $D_{\boldsymbol{k}}^{\prime}$ denote the complementary closed region. Let a point $\alpha_{k}$ be chosen arbitrarily interior to $D_{\boldsymbol{k}}^{\prime}, k=0,1,2, \cdots, \nu$.

Let the function $f(z)$ be given continuous on $C$, analytic in the interior points of $C . A$ necessary and sufficient condition that $f(z)$ be of class $L(p, \alpha)$ for $0<\alpha$ $<1$ or of class $Z_{p}$ for $\alpha=1$ on all $C_{k}$ is that there exist rational functions $R_{n}(z)$ of respective degrees $(\nu+1) n$ having in each point $\alpha_{k}$ no pole of order greater than $n$ and having no other poles, such that $(n>0)$

$$
\left|f(z)-R_{n}(z)\right| \leqq A / n^{p+\alpha}, \quad z \text { on } C \text {. }
$$

If a sequence of functions $F_{n}(z)$ analytic in a region $D$ containing $C$ satisfies the inequality

$$
\left|F_{n}(z)\right| \leqq A_{1} R^{n}, \quad z \text { in } D,
$$

and if for some function $f(z)$ defined on $C$ we have $(n>0)$

$$
\left|f(z)-F_{n}(z)\right| \leqq A_{2} / n^{p+\alpha}, \quad z \text { on } C,
$$

then on each $C_{k}$ the function $f(z)$ is of class $L(p, \alpha)$ for $0<\alpha<1$ or of class $Z_{p}$ for $\alpha=1$. Moreover, each of the $\nu+1$ components $F_{k n}(z), k=0,1,2, \cdots, \nu$, of $F_{n}(z)$ satisfies an inequality $(n>0)$

$$
\left|f_{k}(z)-F_{k n}(z)\right| \leqq A_{3} / n^{p+\alpha}, \quad z \text { on } C,
$$

where $f_{k}(z)$ is the corresponding component of $f(z)$.

In particular if the functions $F_{n}(z)$ are rational functions of $z$ of respective degrees $n$ with no limit point of poles on $C$, then (28) implies (27) in a suitable region $D$.

A function $f(z)$ analytic interior to $C$ and continuous on $C$ can be split into $\nu+1$ components by the equations (all integrals are to be taken in the positive sense with respect to $C$ )

$$
f(z) \equiv \sum_{k=0}^{\nu} f_{k}(z), \quad f_{k}(z) \equiv \frac{1}{2 \pi i} \int_{C_{k}} \frac{f(t) d t}{t-z} ;
$$

the second of equations (30) is valid for $z$ interior to $C$, and defines $f_{k}(z)$ throughout $D_{k}$; it will be noted that $f_{k}(z)$ is analytic at every point of $D_{k}$, 
and $f_{k}(\infty)=0$ for $k>0$; the first of equations (30), valid for $z$ interior to $C$, then defines $f_{k}(z)$ also on $C_{k}$, so $f_{k}(z)$ is continuous on the closure of $D_{k}$ and if $f(z)$ is of class $L(p, \alpha)$ or $Z_{p}$ on $C_{k}$ so also is $f_{k}(z)$. These comments obviously apply to the $\nu+1$ components $R_{k n}(z)$ and $F_{k n}(z)$ of the functions $R_{n}(z)$ and $F_{n}(z)$; each component is analytic at every point of $C$.

Let $f(z)$ of the first part of Theorem 6 be of class $L(p, \alpha)$ or $Z_{p}$ on all $C_{k}$; then $f_{k}(z)$ is continuous in the closure of $D_{k}$ and analytic interior to $D_{k}$; also $f_{k}(z)$ is of class $L(p, \alpha)$ or $Z_{p}$ on $C_{k}$ and can be approximated in the closure of $D_{k}$ by a sequence of rational functions of respective degrees $n$ whose poles lie in $\alpha_{k}$ with degree of approximation $1 / n^{p+\alpha}$. If $R_{n}(z)$ denotes the term-byterm sum of these $\nu+1$ sequences, we have (26). Conversely, if the $R_{n}(z)$ exist so that (26) is valid, the function $f(z)$ is necessarily continuous on $C$ and analytic interior to $C$. From Theorem 4 applied to each $C_{k}$ it follows that $f(z)$ is of class $L(p, \alpha)$ or $Z_{k}$ on each $C_{k}$.

If the functions $F_{n}(z)$ analytic in $D$ satisfy (27) and (28), then Theorem 3 applies at once, and shows that $f(z)$ is of class $L(p, \alpha)$ or $Z_{p}$ on each $C_{k}$. Moreover we have

$$
f_{k}(z)-F_{k n}(z) \equiv \frac{1}{2 \pi i} \int_{C_{k}} \frac{\left[f(t)-F_{n}(t)\right] d t}{t-z}, \quad z \text { in } D_{k},
$$

so on each of the curves $C_{j}$ with $j \neq k$ we have from (28)

$$
\left|f_{k}(z)-F_{k n}(z)\right| \leqq A_{4} / n^{p+\alpha} \text {. }
$$

Inequality $(28)$ can be written

$$
\left|\sum_{k=0}^{\nu}\left[f_{k}(z)-F_{k n}(z)\right]\right| \leqq A_{2} / n^{p+\alpha}, \quad z \text { on } C,
$$

so from (31) we have $(n>0)$

$$
\left|f_{k}(z)-F_{k n}(z)\right| \leqq A_{5} / n^{p+\alpha}, \quad z \text { on } C_{k} ;
$$

thus (32) is valid in the closure of $D_{k}$ and in particular includes (29).

In (32) we have considered that component $F_{k n}(z)$ of $F_{n}(z)$ which is defined in $D_{k}$, not that component $F_{k n}^{*}(z)$ of $F_{n}(z)$ defined by

$$
F_{k n}^{*}(z) \equiv \frac{1}{2 \pi i} \int_{C_{k}} \frac{F_{n}(t) d t}{t-z}, \quad z \text { interior to } D_{k}^{\prime} .
$$

However, at a point $z$ of the $D_{\boldsymbol{k}}^{\prime}$, we have

$$
\begin{aligned}
0= & \sum_{k=0}^{\nu} \frac{1}{2 \pi i} \int_{C_{k}} \frac{F_{n}(t) d t}{t-z}=F_{0 n}(z)+\cdots+F_{k-1, n}(z) \\
& +F_{k n}^{*}(z)+F_{k+1, n}(z)+\cdots+F_{v n}(z),
\end{aligned}
$$


with similar equations for the components $f_{\boldsymbol{k}}^{*}(z)$ of $f(z)$. We define $F_{k n}^{*}(z)$ from the equality of these first and third members so as to be continuous on $C_{k}$, hence continuous in $D_{\boldsymbol{k}}^{\prime}$, and similarly for $f_{\boldsymbol{k}}^{*}(z)$. Thus the behavior of $F_{k n}^{*}(z)$ in $D_{k}^{\prime}$ is readily deduced from (32); we have

$$
\left|f_{k}^{*}(z)-F_{k n}^{*}(z)\right| \leqq A_{6} / n^{p+\alpha}, \quad z \text { in } D_{k}^{\prime} .
$$

The last statement in Theorem 6 follows as in the proof of Theorem 4.

By our method of proof of (29) it is clear that any degree of approximation of $F_{n}(z)$ to $f(z)$ on $C$ assumed in place of (28) implies the same degree of approximation of $F_{k n}(z)$ to $f_{k}(z)$ on $C$; this is in strong contrast to Theorems 2 and 3.

Theorem 6 admits an application precisely as Theorem 3 admits Theorem 5 as application; we omit the proof:

Theorem 7. With the topological notation of Theorem 6 , let $D$ be a region containing $C$ whose closure contains none of the points $\alpha_{k}$. Let the function $f(z)$ be continuous on $C$, analytic interior to $C$. For each positive $M$ let $F_{M}(z)$ be the (or a) function analytic and of modulus not greater than $M$ in $D$ such that the measure of approximation (24) is least. Then a necessary and sufficient condition that $f(z)$ be of class $L(p, \alpha)$ with $0<\alpha<1$ or of class $Z_{p}$ with $\alpha=1$ on $C$ is that (25) be bounded as $M \rightarrow \infty$.

The comments relating to Theorem 5 concerning the boundedness of (25) obviously apply also to Theorem 7 .

\section{REFERENCES}

1. J. L. Walsh, Interpolation and approximation by rational functions, Amer. Math. Soc. Colloquium Publications, vol. 20, New York, 1935.

2. - J. Math. Pures Appl. vol. 31 (1952) pp. 221-244.

3. - Proc. Nat. Acad. Sci. U.S.A. vol. 37 (1951) pp. 821-826.

4. J. L. Walsh and H. Margaret Elliott, Trans. Amer. Math. Soc. vol. 68 (1950) pp. 183203.

5. J. L. Walsh, Bull. Amer. Math. Soc. vol. 52 (1946) pp. 449-453.

6. H. M. Elliott, Proceedings of the American Mathematical Society, to appear.

7. I. E. Block, Duke Math. J. vol. 19 (1952) pp. 367-378.

HARVARD UNIVERSITY,

Cambridge, Mass. 